\title{
Genomic predictions in diploid Actinidia chinensis (kiwifruit)
}

\author{
C.-H. Cheng ${ }^{1}$, P.M. Datson ${ }^{1}$, E. Hilario ${ }^{1}$, C.H. Deng ${ }^{1}$, K.I. Manako ${ }^{1}$, M. McNeilage ${ }^{1}$, M. Bomert ${ }^{1}$ and \\ K. Hoeata ${ }^{2}$ \\ ${ }^{1}$ The New Zealand Institute for Plant \& Food Research Ltd., Mount Albert Research Centre, Auckland, New Zealand \\ ${ }^{2}$ The New Zealand Institute for Plant \& Food Research Ltd., Te Puke Research Centre, Te Puke, New Zealand
}

\section{Summary}

Kiwifruit are dioecious, long-lived, perennial, woody vines. The consequences for kiwifruit breeding include lengthy breeding cycles, large planting areas and selection of male parents by progeny tests, which are expensive and time-consuming. Also, most economically important traits, such as yield, fruit quality and disease resistance, are complex because of their polygenic (quantitative) nature. Genomic selection in kiwifruit has been made possible because of the development of restriction enzymebased genotyping-by-sequencing (GBS), together with new statistical methods for analysing genotypic data. However, information on model robustness and guidelines for model selection are lacking in kiwifruit. In this study, a training population consisted of 88 plants, including four of the parents and 84 seedlings, which were randomly selected from a diploid Actinidia chinensis breeding population. Of these, $\mathbf{7 8}$ were used as a training set and 10 were used as a validation set. Random tagging GBS (rtGBS) libraries were constructed using the restriction enzyme BamHI and sequenced on Illumina ${ }^{\circledR}$ HiSeq2000 platform in Single-end mode. A total of 8372 SNP markers were obtained on all 88 individuals. Three statistical models, RR-BLUP (ridge regression best linear unbiased predictor), GBLUP (genomic best linear unbiased predictor), and LASSO (least absolute shrinkage and selection operator), were used to estimate GEBVs (genomic estimated breeding values) for nine traits. The correlations between predicted GEBVs and observed phenotypic data or breeding values estimated from phenotypic data of the validation set were calculated to measure the reliabilities of the predicted GEBVs. The correlations ranged from 0.48 to 0.91 for red intensity, fruit weight, yield, and fruit firmness at 60,90 and 120 days of storage, whereas negative correlations were obtained for dry matter, soluble solids content and Pseudomonas syringae pv. actinidiae (Psa) resistance. Possible reasons for the negative correlations were discussed. Our results showed that the GBLUP model can be used to predict GEBVs in kiwifruit breeding.

Keywords genotyping-by-sequencing, genomic selection, GEBV, kiwifruit breeding, statistical model

\section{Introduction}

Kiwifruit are dioecious, long-lived, perennial, woody vines. The consequences for kiwifruit breeding include

\section{Significance of this study}

What is already known on this subject?

- Studies of the application of genomic selection in different crops, for example, maize, sugarcane, and apple, have shown the potential of applying GEBV for selection in breeding programmes. However, for kiwifruit there is no information on model robustness and no guidelines for model selection. Genomic selection in kiwifruit has been made possible because of the development of restriction enzyme-based genotyping-by-sequencing (GBS) together with new statistical methods for analysing genotypic data.

What are the new findings?

- Our findings were genomic selection can be applied to kiwifruit breeding and the GBLUP model can be used in our kiwifruit breeding programme.

\section{What is the expected impact on horticulture?}

- Kiwifruit breeding could benefit from genomic selection to overcome lengthy kiwifruit breeding cycles, to reduce planting areas, and to improve accuracy of selection and efficiency in breeding.

lengthy breeding cycles, large planting areas and selection of male parents by progeny tests, which are expensive and time-consuming. The use of molecular markers as aids to kiwifruit selection has been actively studied for several decades and markers useful for selection of traits controlled by a small number of "major" genes have been available for a number of years (Testolin and Cipriani, 2016). For example, markers have been developed in Actinidia chinensis that target the sex chromosomes and allow identification of male and female plants as seedlings (Gill et al., 1998). However, most economically important traits, such as yield, fruit quality and disease resistance, are complex because of their polygenic (quantitative) nature (Cheng et al., 2004; Cheng, 2014). Genomic selection (GS) is able to capture these "minor" gene effects (Meuwissen et al., 2001) through prediction of breeding value (e.g., genomic estimated breeding value (GEBV)) of individuals by joint analysis of all markers on a population attempting to explain the total genetic variance with dense genome-wide marker coverage through summing marker effects. Studies of the application of GS in different crops, for example, maize (Owens et al., 2014), sugarcane (Gouy et al., 2013), and apple (Kumar et al., 2012) have shown the potential of applying GEBV for selection in breeding programmes. GS in kiwifruit has been made possible because of the development of restriction enzyme-based genotyping-by-sequencing (GBS) together with new statistical methods for analysing genotypic data. A further modification of the GBS 
method, random tagging genotyping by sequencing (rtGBS) has been developed and tested in kiwifruit (Hilario, 2015), where the library is prepared from amplified gDNA tagged with a random hexamer linked to the common GBS oligonucleotide instead of intact gDNA.

Genomic selection allows breeders to use GEBVs to select an individual without phenotypic data. Thus, in kiwifruit, breeders can select male parents without lengthy progeny testing. By shifting from phenotype-based selection to genotype-based selection, breeders can discard many vines at the seedling stage, thereby reducing planting areas significantly and allowing more detailed evaluation of valuable seedlings. Furthermore, allowing breeders to select on predictions rather than observations should result in much improved genetic gains, accuracy of selection and efficiency in breeding.

The basic process of GS is the calculation of GEBVs for individuals having only genotypic data employing a model that was "trained" from individuals with both phenotypic and genotypic data (Meuwissen et al., 2001). The population of individuals with both phenotypic and genotypic data is known as the "training population" as is used to estimated model parameters. Many statistical models with different assumptions about the marker effect distribution have been developed to predict breeding values in plants (Heslot et al., 2012). However, for kiwifruit there is no information on model robustness and no guidelines for model selection. Our aim in this study was to test the accuracy of three statistical models for estimating GEBVs of diploid A. chinensis vines and to identify a robust model to be applied in our kiwifruit breeding programme.

\section{Materials and methods}

\section{Plant material}

The training population consisted of 88 vines, four of the parents (Females A and B, and Males 2 and 5) and 84 seedlings which were randomly selected from a diploid A. chinensis breeding population designed to breed kiwifruit with red-centred flesh. The breeding population comprised a disconnected mating factorial, with $2 \times 6$ and $2 \times 2$ subfactorials and a total of 16 full-sib families. It was planted in 2007 at Te Puke Research Centre, the New Zealand Institute for Plant \& Food Research Ltd. (PFR), New Zealand (37 $50^{\prime}$ 'S; $176^{\circ} 18^{\prime} \mathrm{E}$ ). Four female parents (A, B, C and D) and 8 male parents (numbered from 1 to 8 ) were selected, based on progeny testing results from previous studies (Cheng et al., 2004, 2007). Females A and B and Males 7-8 were selected from a germplasm accession, the females of which had fruit with red flesh at their centres, high dry matter (DM) but a short storage life, while Females C and D and Males 1-6 were selected from a germplasm accession, the females of which had fruit with a long storage life and moderate DM but no indication of red flesh. Full-sib families between red parents or between non-red parents were not produced (Table 1). Coefficients of coancestry $\left(f_{i j}\right)$, calculated in ASReml-R (Gilmour et al., 2009), showed that some parents of the populations shared copies of the same ancestral allele. The trial was set out in a completely randomised design with 10 replicates. The seedlings were spaced at $0.6 \mathrm{~m}$ within row and $4 \mathrm{~m}$ between rows on a T-bar trellis structure. Standard cultivation methods for kiwifruit were followed, but vines were not thinned or sprayed.

Phenotypic data were collected for five fruit characters in the 2011 fruit season: fruit weight (FW), yield, DM, soluble solid content (SSC) at eating ripe and red intensity score (Red). Breeding values (BVs) were estimated for fruit firmness (FF) at 60, 90 and 120 days (d) of storage from phenotypic data from a storage trial (Cheng and Day, 2013). Also, BVs of resistance to Psa (Pseudomonas syringae pv. actinidiae) were estimated, as per Cheng (2014). BVs of male parents were also calculated for nine traits.

\section{GBS genotyping}

High-quality total DNA was extracted for the 88 vines using a Qiagen DNeasy plant mini kit following the manufacturer's instructions. rtGBS (a further improvement of the GBS method) libraries were prepared following the protocol of Hilario (2015). We selected the restriction enzyme BamHI based on the predicted number of cutting sites of this enzyme in the diploid $A$. chinensis genome, and the libraries were individually amplified and analysed by agarose gel electrophoresis to verify that they were successfully prepared, before amplicons were pooled prior to sequencing (Hilario, 2015). The prepared rtGBS libraries were sequenced on Illumina ${ }^{\circledR}$ HiSeq2000 platform in Singleend (SE) mode in three lanes at the Australian Genome Research Facility (AGRF).

The data output was 119 to 169 million reads per lane with a read length of 100 nucleotides. The sequencing quality

TABLE 1. Disconnected mating design used in the breeding population and the coefficients of coancestry between female and male Actinidia chinensis parents.

\begin{tabular}{|c|c|c|c|c|c|c|c|c|}
\hline & \multicolumn{4}{|c|}{ Disconnected mating design } & \multicolumn{4}{|c|}{ Coefficients of coancestry } \\
\hline & \multicolumn{2}{|c|}{ Red } & \multicolumn{2}{|c|}{ Non-red } & \multicolumn{2}{|c|}{ Red } & \multicolumn{2}{|c|}{ Non-red } \\
\hline & Female A & Female B & Female C & Female D & Female A & Female B & Female C & Female D \\
\hline \multicolumn{9}{|l|}{ Non-red } \\
\hline Male 1 & $x$ & $x$ & & & 0 & 0 & 0.188 & 0 \\
\hline Male 2 & $x$ & $x$ & & & 0 & 0 & 0.188 & 0 \\
\hline Male 3 & $x$ & $x$ & & & 0 & 0 & 0.188 & 0 \\
\hline Male 4 & $x$ & $x$ & & & 0 & 0 & 0.188 & 0 \\
\hline Male 5 & $x$ & $x$ & & & 0 & 0 & 0.188 & 0 \\
\hline Male 6 & $x$ & $x$ & & & 0 & 0 & 0 & 0 \\
\hline \multicolumn{9}{|l|}{ Red } \\
\hline Male 7 & & & $x$ & $x$ & 0.25 & 0.25 & 0 & 0 \\
\hline Male 8 & & & $x$ & $x$ & 0.25 & 0.625 & 0 & 0 \\
\hline
\end{tabular}




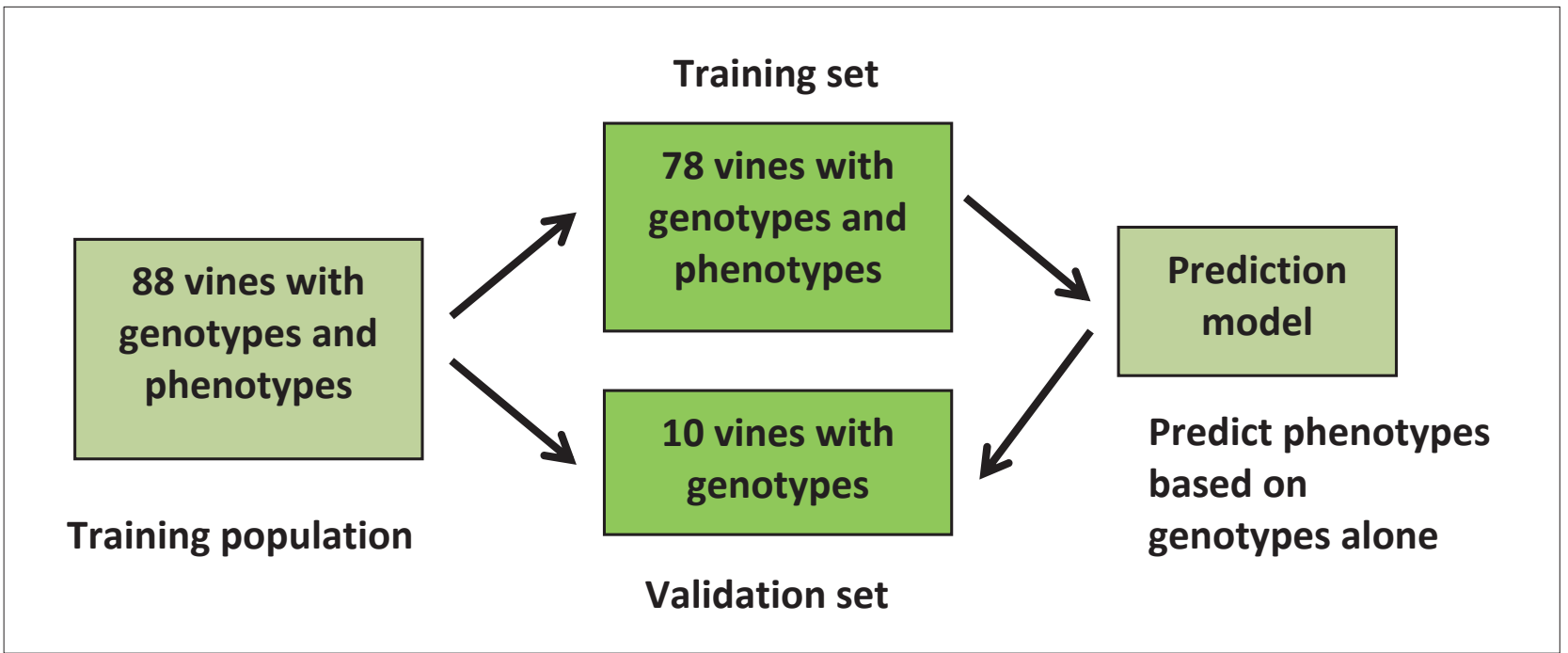

FIGURE 1. Diagram of genomic selection in diploid Actinidia chinensis breeding vines.

was checked using FastQC (Andrews, 2010). No read was discarded for low quality based on the FastQC reports. About $91 \%$ to $96 \%$ acceptable reads (starting with recognisable barcode followed by BamHI cutsite) in each lane were used for SNP detection. Tag sequences of 64 nucleotides were extracted from the acceptable reads and mapped against the 'Hong Yang' genome (Huang et al., 2013), using Bowtie2 (Langmead and Salzberg, 2012). The genotyping analysis was performed using the TASSEL-GBS pipeline (Glaubitz et al., 2014) in TASSEL package (Bradbury et al., 2007), based on the mapping results. The raw SNP sites were further filtered, using GBSHapMapFiltersPlugin with a threshold of minimum site coverage 1.0 and minimum taxon coverage 0.1 . As a result, 40,384 raw SNP sites were detected. A set of 8372 reliable SNP markers were obtained for all 88 individuals after filtering based on no missing data in any of the samples. SNPs were coded as 0 (homozygous state), 1 (heterozygous state) or 2 (alternative homozygous state), and a matrix of markers versus individuals was made.

\section{Statistical models for genomic prediction}

Three statistical models, RR-BLUP (ridge regression best linear unbiased predictor), GBLUP (genomic best linear unbiased predictor), and LASSO (least absolute shrinkage and selection operator), were used to estimate GEBVs for nine traits (Goddard and Hayes, 2007; Habier et al., 2007; Li and Sillanpaa, 2012), using an R package (R Development Core Team, 2008).

In the study, 78 vines were randomly selected as a training set, while 10 were used as a validation set (Figure 1). Genotypic and phenotypic data of 78 vines (the training set) were used to create the prediction models. The models were then used to predict GEBVs of the remaining 10 vines (the validation set), using genotypic data only. The correlations between observed phenotypic data or breeding values (BVs) estimated from phenotypic data and predicted GEBVs of the validation set were calculated to measure the accuracies of the predicted GEBVs, using an R package (R Development Core Team, 2008).

\section{Results and discussion}

Predicted GEBVs from different models for the same trait were highly correlated (Table 2). Previous empirical studies that investigated the effect of predictive parametric models on the accuracy of the phenotype prediction showed that most models performed almost identically, especially for polygenic traits (Daetwyler et al., 2013; Howard et al., 2014). However, the GBLUP model has the advantages of relative

TABLE 2. Correlations between genomic estimated breeding values (GEBVs) and observed phenotypic data or breeding values (BVs) of the validation set for three prediction models.

\begin{tabular}{|c|c|c|c|}
\hline & GBLUP2 $^{2}$ & RR-BLUP2 & LASSO $^{2}$ \\
\hline Red intensity & 0.592 & 0.591 & 0.632 \\
\hline Fruit weight & 0.527 & 0.529 & 0.524 \\
\hline Yield & 0.566 & 0.563 & 0.478 \\
\hline Fruit firmness at 60 days $^{1}$ & 0.910 & 0.913 & 0.902 \\
\hline Fruit firmness at 90 days ${ }^{1}$ & 0.558 & 0.563 & 0.578 \\
\hline Fruit firmness at 120 days $^{1}$ & 0.852 & 0.855 & 0.910 \\
\hline Dry matter & -0.448 & -0.438 & -0.429 \\
\hline Soluble solids content & -0.318 & -0.312 & -0.215 \\
\hline Psa resistance ${ }^{1}$ & -0.372 & -0.367 & -0.279 \\
\hline
\end{tabular}

1 BVs calculated from phenotypic data; Psa: abbreviation of Pseudomonas syringae pv. actinidiae.

${ }^{2}$ GBLUP, RR-BLUP and LASSO: abbreviations of genomic best linear unbiased predictor, ridge regression best linear unbiased predictor and least absolute shrinkage and selection operator, respectively. 
simplicity, requirement for limited computing time, and wellknown optimality properties of mixed models for selection (Fernando and Gianola, 1986).

Correlations between observed phenotypic data or BVs estimated from phenotypic data and predicted GEBVs of the validation set were medium-to-high for Red, FW, yield, and FF at 60, 90 and 120 days of storage, although the training population was very small (Table 2). The results indicated that GS can be applied to kiwifruit breeding. Thus, two features of kiwifruit biology, dioecy and long generation times, which impose long breeding cycles in phenotypic recurrent selection regimes, would be less significant under GS, particularly for accuracy and efficiency of selection for male parents. Moreover, GS will allow breeders to discard many vines at the seedling stage, which can reduce planting areas significantly and allow proper evaluation of valuable seedlings.

However, negative correlations were obtained for DM, SSC and Psa resistance. The differences of prediction accuracy for various traits could be partially due to a different genetic architecture (Hayes et al., 2009). The authors pointed out that a large training population would be required to estimate these effects accurately if very many QTLs (quantitative trait locus) with very small effects are contributing to variation in the trait. Our results might imply that those traits (DM, SSC and Psa resistance) with negative correlations might be controlled by a large number of QTLs with small effects. Thus, the small number of individuals in the training data set could be an important reason for the negative correlations. Generally, as sample size increases prediction accuracy also increases even though it is crucial that other influences be also considered (Heffner et al., 2011; Desta and Ortiz, 2014). Also, GS relies on the assumption that all QTLs tend to be in strong linkage disequilibrium (LD) with at least one marker. Thus, our negative correlations could be caused by missing SNPs or SNP errors that are close to QTLs with major effects. Increasing marker density and quality control of SNPs can ensure the conservation of marker-QTL associations and achieves a high prediction accuracy (Heffner et al., 2009; Forneris et al., 2015). Further work is needed to improve GBS genotyping with a large training population of diploid $A$. chinensis to validate the models and to gain a high prediction accuracy, particularly for those traits with negative correlations. Additionally, many kiwifruit species are polyploid, including important breeding populations of tetraploid A. chinensis, A. arguta and hexaploid $A$. deliciosa. Thus, further work is needed to apply GS to polyploid kiwifruit populations.

\section{Conclusions}

Overall the results indicated that GS can be applied to kiwifruit breeding, paving the way for further investigations into the potential of GS for quantitative traits. Thus, kiwifruit breeding could benefit from GS to overcome lengthy kiwifruit breeding cycles, to reduce planting areas, and to improve accuracy of selection and efficiency in breeding. Although predicted GEBVs from different models for the same trait were highly correlated, we recommend using the GBLUP model in our kiwifruit breeding programme because of its advantages of relative simplicity, requirement for limited computing time, and the well-known optimality properties of mixed models for selection. Further work is needed to improve GBS genotyping and phenotyping in kiwifruit and also to enable the application of GS to polyploid kiwifruit populations.

\section{Acknowledgments}

This research was funded by The New Zealand Institute for Plant \& Food Research Ltd. (PFR). We thank Drs. Ross Ferguson, Vincent Bus and Satish Kumar for valuable comments on the paper, and the staff at the Te Puke Research Centre for their assistance with this research.

\section{References}

Andrews, S. (2010). FastQC: a quality control tool for high throughput sequence data. http://www.bioinformatics.babraham. ac.uk/projects/fastqc/.

Bradbury, P.J., Zhang, Z., Kroon, D.E., Casstevens, T.M., Ramdoss, Y., and Buckler, E.S. (2007). TASSEL: software for association mapping of complex traits in diverse samples. Bioinformatics 23, 2633-2635. https://doi.org/10.1093/bioinformatics/btm308.

Cheng, C.H. (2014). Inheritance of resistance to Pseudomonas syringae pv. actinidiae and genetic correlations with fruit characters in a diploid Actinidia chinensis (kiwifruit) population. Euphytica 198, 305-315. https://doi.org/10.1007/s10681-014-1107-3.

Cheng, C.H., and Day, S. (2013). Genetic study on fruit storage life in a disconnected factorial mating population of Actinidia chinensis (kiwifruit). Acta Hortic. 976, 377-382. https://doi.org/10.17660/ ActaHortic.2013.976.51.

Cheng, C.H., Seal, A.G., Boldingh, H.L., Marsh, K.B., MacRae, E.A., Murphy, S.J., and Ferguson, A.R. (2004). Inheritance of taste characters and fruit size and number in a diploid Actinidia chinensis (kiwifruit) population. Euphytica 138, 185-195. https://doi. org/10.1023/B:EUPH.0000046802.28347.41.

Cheng, C.H., Seal, A.G., Murphy, S.J., and Lowe, R.G. (2007). Red-fleshed kiwifruit (Actinidia chinensis) breeding in New Zealand. Acta Hortic. 753, 139-146. https://doi.org/10.17660/ActaHortic.2007.753.14.

Daetwyler, H.D., Calus, M.P.L., Pong-Wong, R., de los Campos, G., and Hickey, J.M. (2013). Genomic prediction in animals and plants: Simulation of data, validation, reporting, and benchmarking. Genetics 193, 347-373. https://doi.org/10.1534/genetics.112.147983.

Desta, Z.A., and Ortiz, R. (2014). Genomic selection: genome-wide prediction in plant improvement. Trends Plant Sci. 19, 592-601. https://doi.org/10.1016/j.tplants.2014.05.006.

Fernando, R.L., and Gianola, D. (1986). Optimal properties of the conditional mean as a selection criterion. Theor. Appl. Genet. 72, 822-825. https://doi.org/10.1007/BF00266552.

Forneris, N.S., Legarra, A., Vitezica, Z.G., Tsuruta, S., Aguilar, I., Misztal, I., and Cantet, R.J.C. (2015). Quality control of genotypes using heritability estimates of gene content at the marker. Genetics 199, 675-686. https://doi.org/10.1534/genetics.114.173559.

Gill, G.P., Harvey, C.F., Gardner, R.C., and Fraser, L.G. (1998). Development of sex-linked PCR markers for gender identification in Actinidia. Theor. Appl. Genet. 97, 439-445. https://doi.org/10.1007/ s001220050914.

Gilmour, A.R., Gogel, B.J., Cullis, B.R., and Thompson, R. (2009). ASReml User Guide, Release 3.0 (Hemel Hempstead, UK: VSN International Ltd.).

Glaubitz, J.C., Casstevens, T.M., Lu, F., Harriman, J., Elshire, R.J., Sun, Q., and Buckler, E.S. (2014). TASSEL-GBS: a high capacity genotyping by sequencing analysis pipeline. PLoS One 9, e90346. https://doi. org/10.1371/journal.pone.0090346.

Goddard, M.E., and Hayes, B.J. (2007). Genomic selection. J. Anim. Breed. Genet. 124, 323-330. https://doi.org/10.1111/j.14390388.2007.00702.x.

Gouy, M., Rousselle, Y., Bastianelli, D., Lecomte, P., Bonnal, L., Roques, D., Efile, J.C., Rocher, S., Daugrois, J., Toubi, L., Nabeneza, S., Hervouet, C., Telismart, H., Denis, M., Thong-Chane, A., Glaszmann, J.C., Hoarau, 
J.Y., Nibouche, S., and Costet, L. (2013). Experimental assessment of the accuracy of genomic selection in sugarcane. Theor. Appl. Genet. 126, 2575-2586. https://doi.org/10.1007/s00122-013-2156-z.

Habier, D., Fernando, R.L., and Dekkers, J.C.M. (2007). The impact of genetic relationship information on genome-assisted breeding values. Genetics 177, 2389-2397. https://doi.org/10.1534/ genetics.107.081190.

Hayes, B.J., Visscher, P.M., and Goddard, M.E. (2009). Increased accuracy of artificial selection by using the realized relationship matrix. Genetics Res. 91, 47-60. https://doi.org/10.1017/ S0016672308009981.

Heffner, E.L., Sorrells, M.E., and Jannink, J.L. (2009). Genomic selection for crop improvement. Crop Sci. 49, 1-12. https://doi. org/10.2135/cropsci2008.08.0512.

Heffner, E.L., Jannink, J.L., and Sorrells, M.E. (2011). Genomic selection accuracy using multifamily prediction models in a wheat breeding program. Plant Genome 4, 65-75. https://doi. org/10.3835/plantgenome2010.12.0029.

Heslot, N., Yang, H.-P., Sorrells, M.E., and Jannink, J.-L. (2012) Genomic selection in plant breeding: A comparison of models. Crop Sci. 52, 146-160. https://doi.org/10.2135/cropsci2011.09.0297.

Hilario, E. (2015). The restriction enzyme target approach to genotyping by sequencing (GBS). Methods Mol. Biol. 1245, 271-279. https://doi.org/10.1007/978-1-4939-1966-6_20.

Howard, R., Carriquiry, A.L., and Beavis, W.D. (2014). Parametric and nonparametric statistical methods for genomic selection of traits with additive and epistatic genetic architectures. G3-Genes Genomes Genetics 4, 1027-1046. https://doi.org/10.1534/g3.114.010298.

Huang, S., Ding, J., Deng, D., Tang, W., Sun, H., Liu, D., Zhang, L., Niu, X., Zhang, X., Meng, M., Yu, J., Liu, J., Han, Y., Shi, W., Zhang, D., Cao, S., Wei, Z., Cui, Y., Xia, Y., Zeng, H., Bao, K., Lin, L., Min, Y., Zhang, H., Miao, M., Tang, X., Zhu, Y., Sui, Y., Li, G., Sun, H., Yue, J., Sun, J., Liu, F., Zhou, L., Lei, L., Zheng, X., Liu, M., Huang, L., Song, J., Xu, C., Li, J., Ye, K., Zhong, S., Lu, B.-R., He, G., Xiao, F., Wang, H.-L., Zheng, H., Fei, Z., and Liu, Y. (2013). Draft genome of the kiwifruit Actinidia chinensis. Nat. Commun. 4, 2640. https://doi.org/10.1038/ncomms3640.

Kumar, S., Chagné, D., Bink, M.C.A.M., Volz, R.K., Whitworth, C., and Carlisle, C. (2012). Genomic selection for fruit quality traits in apple (Malus $\times$ domestica Borkh.). PLoS ONE 7, e36674. https://doi. org/10.1371/journal.pone.0036674.

Langmead, B., and Salzberg, S.L. (2012). Fast gapped-read alignment with Bowtie 2. Nat. Methods 9, 357-359. https://doi.org/10.1038/ nmeth.1923.

Li, Z.T., and Sillanpaa, M.J. (2012). Overview of LASSO-related penalized regression methods for quantitative trait mapping and genomic selection. Theor. Appl. Genet. 125, 419-435. https://doi. org/10.1007/s00122-012-1892-9.

Meuwissen, T.H.E., Hayes, B.J., and Goddard, M.E. (2001). Prediction of total genetic value using genome-wide dense marker maps. Genetics 157, 1819-1829.

Owens, B.F., Lipka, A.E., Magallanes-Lundback, M., Tiede, T., Diepenbrock, C.H., Kandianis, C.B., Kim, E., Cepela, J., MateosHernandez, M., Buell, C.R., Buckler, E.S., DellaPenna, D., Gore, M.A., and Rocheford, T. (2014). A foundation for provitamin A biofortification of maize: Genome-wide association and genomic prediction models of carotenoid levels. Genetics 198, 1699-1780. https://doi.org/10.1534/genetics.114.169979.

R Foundation for Statistical Computing. (2008). R: a language and environment for statistical computing (Vienna, Austria: R Foundation for Statistical Computing).

Testolin, R., and Cipriani, G. (2016). Markers, maps and markerassisted selection. In The Kiwifruit Genome, R. Testolin, H.-W. Huang, and R.A. Ferguson, eds. (Cham, Switzerland: Springer International Publishing), p. 249-257. https://doi.org/10.1007/978-3-31932274-2 7 .

Received: May 26, 2017

Accepted: Feb. 13, 2019

Addresses of authors:

C.-H. Cheng ${ }^{1, *}$, Paul M. Datson ${ }^{1}$, Elena Hilario ${ }^{1}$,

Cecilia H. Deng ${ }^{1}$, Kelvina I. Manako ${ }^{1}$, Mark McNeilage ${ }^{1}$,

Maud Bomert ${ }^{1}$ and Kirsten Hoeata ${ }^{2}$

${ }^{1}$ The New Zealand Institute for Plant \& Food Research Ltd., Mount Albert Research Centre, Private Bag 92 169,

Auckland, New Zealand

2 The New Zealand Institute for Plant \& Food Research Ltd., Te Puke Research Centre, 412 No 1 Road, RD2, Te Puke, 3182, New Zealand

* Corresponding author;

E-mail: canhong.cheng@plantandfood.co.nz 